\title{
Aplicação das normas L1 e Lo em redes altimétricas: identificação de outliers e construção do modelo estocástico
}

\author{
L1 and Lo norms adjustment in leveling networks: \\ outlier identification and stochastic modeling
}

\author{
Stefano Sampaio Suraci* \\ Leonardo Castro de Oliveira**
}

Recebido 30 de agosto de 2019; aceito 2 de novembro de 2019

\section{Resumo}

Nesse artigo, aplicações da minimização da norma L1 (ML1) e da norma Lœ (ML $\infty)$ na estimação de redes altimétricas foram investigadas. Redes de nivelamento simuladas pela Técnica de Monte Carlo e dados reais da rede brasileira de nivelamento foram empregados nos experimentos. Na identificação de outliers pela ML1, foi verificado que o ajustamento com pesos unitários apresentou condições vantajosas em relação ao modelo estocástico usual dos pesos proporcionais ao inverso do comprimento das linhas de nivelamento. O Classificador VL1, que estipula um Valor de Corte para os resíduos do ajustamento pela ML1 a partir do qual a respectiva observação passa a ser classificada como outlier, foi proposto. A taxa de sucesso dele na identificação de outliers foi superior à do procedimento data snooping iterativo em cenários de geometria da rede deficiente. Já a aplicação da ML $\infty$ investigada é posterior ao tratamento de outliers. Um modelo estocástico alternativo para ajustamento da rede pelo MMQ que aproveitou a característica de minimização do máximo resíduo absoluto da rede no ajustamento pela $\mathrm{ML} \infty$ foi analisado. Além dessa minimização, o ajustamento da rede pelo MMQ com o modelo

* Instituto Militar de Engenharia, Brasil, correio eletrônico: stefano@ime.eb.br.

ORCID: https://orcid.org/0000-0002-4453-9273.

**Instituto Militar de Engenharia, Brasil, correio eletrônico: leonardo@ime.eb.br.

ORCID: https://orcid.org/0000-0001-5290-5029. 
alternativo gerou, na significativa maioria dos casos, resíduos e precisão desses e dos parâmetros estimados mais homogêneos, com menor desvio padrão, que aqueles com o modelo estocástico usual. Todos os resultados são especialmente relevantes para o caso de redes altimétricas.

Palavras chaves: normas L1e Lœ, modelo estocástico, Monte Carlo, altimetria.

\section{Abstract}

In this paper, applications of L1 norm minimization (ML1) and of L $\infty$ norm minimization $(\mathrm{ML} \infty)$ in the estimation of leveling networks were investigated. Leveling networks simulated by the Monte Carlo technique and real data from the Brazilian leveling network were employed in the experiments. In the identification of outliers by ML1, it was verified that the adjustment with unit weights presented advantageous conditions in relation to the usual stochastic model of weights of observations as proportional to the inverse of the length of the leveling lines. The Classificador VL1, which stipulates a cut-off value for the residuals of the adjustment by ML1 from which the respective observation is classified as outlier, was proposed. Its success rate in identifying outliers was higher than that of the iterative data snooping procedure in poor network geometry scenarios. The application investigated for ML $\infty$ is after the treatment of outliers. An alternative stochastic model for network adjustment by Least Squares (LS) that took advantage of the characteristic of minimization of the maximum absolute residual of the network in the adjustment by ML $\infty$ was analyzed. In addition to this minimization, the adjustment of the network by LS with the proposed model generated, in most cases, residuals and precision of these and of the estimated parameters more homogeneous, with lower standard deviation, than those with the usual stochastic model. All results are especially relevant for the case of altimetric networks.

Palabras clave: L1 and Lo norms, stochastic model, Monte Carlo, leveling networks.

\section{Introdução}

A altimetria tem recebido especial atenção atualmente no contexto dos sistemas de referência. Prova disto é a Resolução 1/2015 (IAG, 2015) da International Association of Geodesy (IAG), que definiu o International Height Reference System (IHRS), com geoide único e adoção de números geopotenciais, reconhecendo a necessidade de um sistema global de alta acurácia para investigação de mudanças na Terra, como a elevação dos níveis dos oceanos.

Nesse trabalho, aplicações da minimização das normas L1 e L $\infty$ no contexto da estimação de redes altimétricas foram investigadas, mais especificamente nas etapas de identificação de outliers e na definição do modelo estocástico para ajustamento 
final da rede pelo Método dos Mínimos Quadrados (MMQ), respectivamente. Com isso, espera-se que seus resultados contribuam para a definição de metodologias para a implantação de redes altimétricas a nível nacional e internacional.

Nos subitens a seguir, a revisão teórica e as situações específicas que originaram as hipóteses dessa pesquisa foram evidenciadas.

\section{Normas L1, L2 e L $\infty$ no ajustamento de observações geodésicas}

$\mathrm{Na}$ estimação de redes geodésicas, o número de observações é superior ao de incógnitas. Os inevitáveis erros de medida tornam o sistema inconsistente (Gemael et al., 2015). O ajustamento da rede é usualmente realizado pelo MMQ, também referenciado por minimização da norma L2 dos resíduos.

O MMQ minimiza a soma dos quadrados dos resíduos $\mathbf{v}$ (diferença entre valores ajustados e observados), ponderados pela matriz dos pesos das observações $\mathbf{P}$ (Equação 1). Seus resultados são os de mínima variância para os parâmetros estimados e de máxima verossimilhança, essa sob a premissa de ocorrência de somente erros aleatórios normalmente distribuídos nas observações (Ghilani, 2010).

$$
M M Q: \min \left(v^{T} P v\right)
$$

Apesar de menos conhecida que o MMQ, a minimização da norma L1 (ML1) também já foi testada no ajustamento de redes geodésicas por diversos autores, como (Marshall \& Bethel, 1996), (Amiri-Simkooei, 2003) e (Yetkin \& Inal, 2011). Para observações independentes, sendo p o vetor dos pesos, expresso pelos elementos da diagonal principal de $\mathbf{P}$, ela minimiza a soma dos resíduos absolutos ponderados por p (Equação 2). O método SIMPLEx de programação linear (Dantzig, 1963) é o mais utilizado na literatura geodésica para solução da ML1.

$$
\operatorname{ML1}: \min \left(p^{T}|v|\right)
$$

Outra minimização de norma vetorial, bem menos explorada na estimação de redes geodésicas, envolve o ajustamento pela minimização da norma L $\infty$ (ML $\infty$ ). Por analogia com o caso de regressão polinomial de (Mason \& Handscomb, 2003), o ajustamento pela MLœ pode ser entendido como a minimização do máximo resíduo absoluto ponderado pelo peso da respectiva observação (Equação 3), sendo m o número de observações. (Ebong, 1986) foi o único registro encontrado de aplicação da norma L $\infty$ em redes geodésicas. Uma formulação detalhada da solução da ML $\infty$ por programação linear foi apresentada em (Suraci \& Oliveira, 2019a), artigo 1 (de 4) 
produzido no contexto da dissertação de mestrado (Suraci, 2018) a que o presente artigo se refere.

$$
M L \infty: \min \left(\operatorname{máx}\left(p_{i}^{*}\left|v_{i}\right|\right)\right), 1 \leq i \leq m
$$

\section{Identificação de outliers em redes geodésicas e a aplicação da ML1}

Apesar de o MMQ ser uma técnica estatística poderosa para o ajustamento de observações, ele demanda a inexistência de outliers para produzir resultados confiáveis (Yetkin \& Inal, 2011). Entretanto, é inviável se garantir a ausência deles na prática dos levantamentos geodésicos.

Nesse trabalho, as observações com magnitude do erro total de medida superior a três vezes o seu respectivo desvio padrão $(\sigma)$ foram consideradas outliers. Uma análise conceitual sobre termos relacionados a outliers, seus significados e aspectos, foi apresentada em (Suraci \& Oliveira, 2019b), artigo 2 (de 4) produzido no contexto da dissertação de mestrado (Suraci, 2018) a que o presente artigo se refere. Nessas duas referências, essa regra adotada para definição de outliers foi intitulada regra dos $3 \sigma$ para o erro total.

Há duas principais abordagens para a identificação de outliers em redes geodésicas: testes estatísticos e métodos robustos. Classificado no primeiro grupo, o procedimento data-snooping, proposto inicialmente por (Baarda, 1968), é um dos melhores métodos para identificação de outliers em redes geodésicas (Rofatto et al., 2017). O data-snooping iterativo (Teunissen, 2006), um procedimento que consiste na aplicação iterativa do data-snooping, é o mais usual e será referido simplesmente por data-snooping (DS) nesse trabalho. Aplicando o DS, (Rofatto et al., 2018) apresentaram recentemente uma relevante inovação na identificação de outliers em redes geodésicas: a aferição de níveis de probabilidade de acerto e dos diferentes tipos de erro via Simulação de Monte Carlo (SMC).

A SMC é um tipo de simulação que se baseia em repetidas amostras aleatórias das variáveis de entrada, permitindo dessa forma uma acurada caracterização da variação dos resultados do modelo a ser estudado (Raychaudhuri, 2008). Quanto maior a quantidade de amostras aleatórias simuladas, mais acurada será a referida caracterização (Rofatto et al., 2018). Sua aplicação tem se tornado usual também na avaliação de métodos de identificação de outliers em redes geodésicas, podendo ser vista, por exemplo, nesse último artigo, em (Klein et al., 2015), em (Hekimoglu \& Erenoglu, 2007) e em (Durdag et al., 2016).

Já no âmbito dos métodos robustos, a ML1 também pode ser aplicada na identificação de outliers. Por ser um método resistente a outliers (Abdelmalek \& Malek, 2008), tende a apresentar resíduos absolutos relativamente maiores para os outliers das redes analisadas. Assim, observações com resíduos absolutos 
relativamente "grandes" podem ser classificadas como outliers. Esse procedimento foi demonstrado por (Amiri-Simkooei, 2003) em redes de nivelamento e trilateração, e por (Yetkin \& Inal, 2011) em redes GNSS. Um exemplo detalhado da questão da classificação de observações como outlier a partir de seu resíduo absoluto relativamente "grande" no ajustamento pela ML1 foi apresentado em (Suraci, 2018).

Outras aplicações da ML1 podem ainda ser vistas em (Marshall \& Bethel, 1996), (Kampmann \& Krause, 1997), (Koch, 1999), (Baselga, 2007), (Khodabandeh \& Amiri-Simkooei, 2011), e (Amiri-Simkooei, 2018).

Diversos outros métodos robustos já foram aplicados em redes geodésicas. Em especial, recentemente, (Koch et al., 2019) apresentaram aplicações de alguns deles, com proposta de uma nova meta-heurística para solução dos mesmos. Vale ressaltar que (Rofatto et al., 2018) colocam os algoritmos meta-heurísticos, junto com a SMC e os algoritmos genéticos, dentre outras, como uma das técnicas cujo potencial ainda não foi totalmente explorado em geodésia.

Entretanto, voltando à identificação de outliers pela ML1, o que seria um resíduo absoluto "grande"? Não há na literatura um critério objetivo estabelecido para classificação de uma observação como outlier a partir de seu respectivo resíduo absoluto no ajuste pela ML1. É razoável visualizar que isto se torna crítico principalmente para outliers de magnitude relativamente menores e para caracterização de uma rede como sem outlier. O Classificador VL1 proposto no corrente artigo estabelece um possível critério a ser adotado.

Ademais, não há uma investigação que valide a escolha do resíduo absoluto como parâmetro de comparação para identificação de outliers. Uma vez que o ajustamento pela ML1 adota determinado modelo estocástico, pode-se imaginar que o resíduo absoluto ponderado seja também um parâmetro viável de comparação. Nesse sentido, a potencial eficácia de um ajustamento com pesos unitários para as observações na identificação de outliers, para o caso do resíduo absoluto ser mesmo o parâmetro a ser analisado é também passível de investigação.

Essa questão foi abordada nos experimentos desse trabalho, sendo intitulada Alternativa de Pesos e de Parâmetro de Comparação para a ML1 (APPC-ML1). A verificação da eficácia de se adotar pesos unitários, juntamente com outros aspectos da ML1, foi abordada em (Suraci et al., 2019), artigo 3 (de 4) produzido no contexto da dissertação de mestrado (Suraci, 2018) a que o presente artigo se refere. Em relação a esse último, a principal contribuição do corrente artigo corresponde à verificação também de Alternativa para o Parâmetro de Comparação e sua integração com a Alternativa de Pesos. Os resultados dessa questão foram aplicados na construção do Classificador VL1.

Para um apropriado entendimento dos experimentos, é importante apresentar ainda o Método das Equações Medianas (MEM). Segundo (Hekimoglu et al., 2011), em redes geodésicas, falhas na identificação de outliers podem ocorrer devido a 
pouca redundância das respectivas observações, independentemente do método utilizado na identificação deles. Assim, foi proposto o MEM: um método para identificação de redundância deficiente na geometria de redes geodésicas, que verifica se um conjunto de outliers é controlável (passível de ser confiavelmente identificado) ou não. Maiores detalhes sobre o MEM no referido artigo.

\section{Modelagem estocástica em redes altimétricas e a aplicação da MLœ}

O modelo estocástico usual em redes altimétricas adota pesos das observações como inversamente proporcionais ao comprimento das respectivas linhas de nivelamento. É baseado em conjecturas cuja garantia de ocorrência na prática dos levantamentos é pouco viável, como a utilização de equipamentos com a mesma precisão e adoção da mesma distância em todas as visadas da rede (Ghilani, 2010), o que justifica a busca por abordagens alternativas para o modelo estocástico de redes altimétricas.

Além disso, em redes de nivelamento o número de injunções normalmente é relativamente baixo. No caso da Rede Altimétrica de Alta Precisão (RAAP) do Sistema Geodésico Brasileiro, por exemplo, há apenas uma injunção para o trecho que atende a maior parte do território. Com o afastamento das referências de nível (RRNN) da injunção, devido à propagação de covariâncias, isso tende a gerar RRNN com precisões muito piores do que outras, como visto em (IBGE, 2018).

Da análise da Equação 3, nota-se que, caso se adotem pesos unitários para as observações, o ajustamento pela MLœ irá acarretar a minimização do máximo resíduo absoluto (MRA) do ajustamento. No presente trabalho, a abordagem alternativa para o modelo estocástico do ajustamento pelo MMQ de redes altimétricas visou minimizar o MRA desse ajustamento, para verificação dos possíveis efeitos na homogeneidade dos resultados. Essa investigação foi intitulada Alternativa de Pesos para o $M M Q(A P-M M Q)$.

Deve ficar claro para o leitor que a aplicação da ML $\infty$ é proposta para uma fase posterior à identificação de outliers na rede, ou seja, em redes livres de outliers. Isso, também, pois a ML $\infty$ tende a distribuir erros de outliers entre as demais observações, o que dificultaria sua aplicação na identificação dos mesmos (Abdelmalek \& Malek, 2008; Suraci \& Oliveira, 2019a).

A proposta e a forma de obtenção desse modelo estocástico alternativo advindo do MRA foram apresentadas em (Suraci \& Oliveira, 2019c), artigo 4 (de 4) produzido no contexto da dissertação de mestrado (Suraci, 2018) a que o presente artigo se refere. Resumidamente, para cálculo do mínimo MRA, um pré-ajuste da rede pela ML $\infty$ com pesos iguais para as observações é aplicado. Em seguida, o modelo estocástico alternativo é obtido iterativamente, incrementando (a cada iteração) o peso de observações com resíduo absoluto maior que o mínimo MRA calculado. As iterações terminam quando nenhum resíduo absoluto dentre as observações da rede for maior que o mínimo MRA, considerando a tolerância estipulada. Em relação ao 
artigo citado, a contribuição do corrente artigo refere-se à análise dos resultados do modelo proposto com aplicação de SMC.

\section{Hipóteses}

A partir do conteúdo abordado na introdução, foram formuladas duas hipóteses, a saber:

- Há cenários de redes altimétricas em que o Classificador VL1 é mais eficaz que o DS na identificação de outliers.

- O ajustamento pelo MMQ de redes altimétricas livres de outliers com modelagem estocástica advinda do MRA gera resultados mais homogêneos que com o modelo usual.

\section{Metodologia}

Conforme visto, as investigações desse trabalho podem ser divididas em três tópicos: a $A P P C-M L 1$, o Classificador VL1 e a $A P-M M Q$, os quais são detalhados na sequência.

\section{APPC-ML1}

As Alternativas de Pesos e de Parâmetro de Comparação para a ML1 foram realizadas de maneira conjunta nos experimentos, pois se desejou investigar qual abordagem para o modelo estocástico e com qual parâmetro de comparação, na identificação de outliers a partir de resultados do ajustamento da rede pela ML1, apresenta condições mais vantajosas para a execução dessa tarefa. Entendeu-se por vantajosa a ocorrência do parâmetro de comparação do outlier como sendo o maior dentre as observações da rede. Caso isto não ocorra, pode-se considerar que houve o indesejado mascaramento do outlier.

Nesse trabalho, com aplicação de SMC, isso foi mensurado em redes altimétricas pelo percentual de ocorrências do parâmetro de comparação do outlier como sendo o maior dentre as observações da rede (PPCOMR), apresentado na Equação 4. Quanto maior o PPCOMR, mais vantajosa a abordagem conjunta avaliada. Similarmente, para cenários de múltiplos outliers, o numerador do PPCOMR computou a quantidade de experimentos em que os valores do parâmetro de comparação dos outliers foram os maiores da rede.

$$
\text { PPCOMR }=\frac{\text { testes em que o parâmetro de comparação do outlier foi o maior da rede }}{\text { total de experimentos }}
$$


O PPCOMR foi avaliado também se adotando pesos unitários com o resíduo absoluto como parâmetro de comparação e pesos usuais com o resíduo absoluto ponderado como parâmetro. Assim, além da abordagem comum na literatura (pesos usuais com resíduo absoluto), duas alternativas foram testadas, totalizando três comparadas. Obviamente, uma quarta opção, que seria se testar pesos unitários com resíduos absolutos ponderados, não se fez necessária, pois ela é equivalente à abordagem alternativa citada de pesos unitários e resíduos absolutos.

\section{O Classificador VL1 proposto}

Já foi visto que o ajustamento pela ML1 é resistente a outliers e, assim, normalmente faz com que os resíduos absolutos desses tendam a ser relativamente maiores. Visando tirar proveito dessa característica, a estratégia do Classificador VL1 é determinar um valor de corte (VC), intitulado VCL1, para o resíduo absoluto de uma observação no ajustamento da rede pela ML1, a partir do qual a mesma passará a ser classificada como outlier. Assim, a primeira etapa da aplicação do Classificador é a construção do VCL1 da rede analisada, caso o mesmo não esteja disponível. A Figura 1 ilustra a aplicação completa do Classificador VL1.

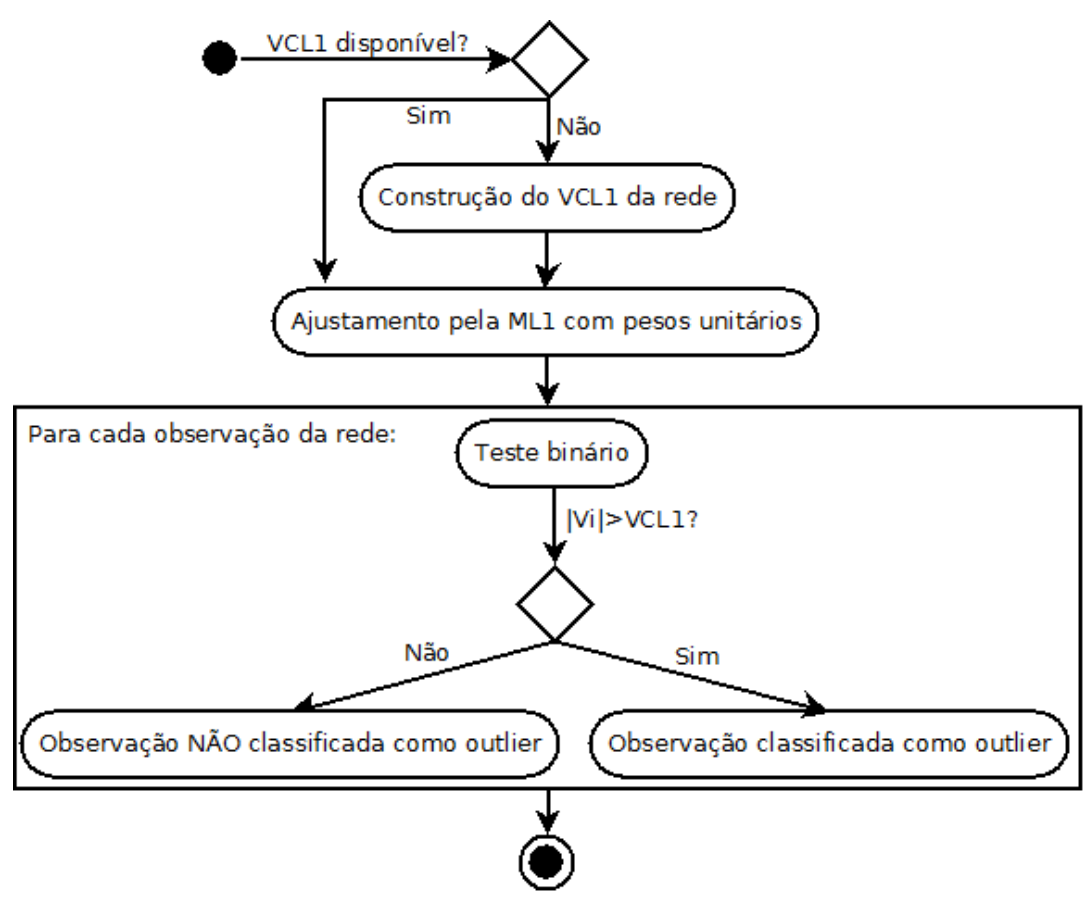

Figura 1. Aplicação do Classificador VL1. 
Após isso, a rede deve ser ajustada pela ML1, adotando-se modelo estocástico com pesos unitários para as observações. Em seguida, vem a decisão binária sobre a classificação como outlier de cada observação da rede, pela comparação de seu respectivo resíduo absoluto $\left|\mathbf{v i}_{\mathbf{i}}\right|$ com o valor de corte VCL1. Caso seja maior, considera-se que a i-ésima observação é um outlier (Equação 5).

$$
\text { se }\left|v_{i}\right|>V C L 1 \text {, a i-ésima observação da rede é um outlier }
$$

Vale esclarecer que a decisão de se utilizar a ML1 com pesos unitários e a confirmação do resíduo absoluto como parâmetro de comparação para o Classificador VL1 está amparada pelos resultados dos experimentos da $A P P C-M L 1$, que são apresentados na sequência desse artigo. Neles se verificou que com essa abordagem conjunta houve maior PPCOMR.

Para construção do valor de corte VCL1 do Classificador VL1 (Figura 2), cenários aleatórios com outlier referentes à rede altimétrica analisada devem ser preparados via SMC. Concluídas as simulações, um $\mathbf{V C}_{\mathbf{k}}$ é estipulado - $\mathbf{k}$ é o número de ordem do VC. Fazendo VCL1 $=\mathbf{V C}_{\mathbf{k}}$, testa-se o comportamento dele para todos os cenários simulados da rede analisada, obtendo-se a respectiva Taxa de Sucesso (TS) (Equação 6). Esse processo é iterativo até que se chegue, por tentativa e erro, a um $\mathbf{V C}_{\mathbf{k}}$ no qual a TS seja máxima, o qual será o valor final para o VCL1 a ser empregado para a identificação de outliers.

$$
T S=\frac{\text { quantidade de classificações corretas }}{\text { total de experimentos }}, 0 \leq T S \leq 1
$$

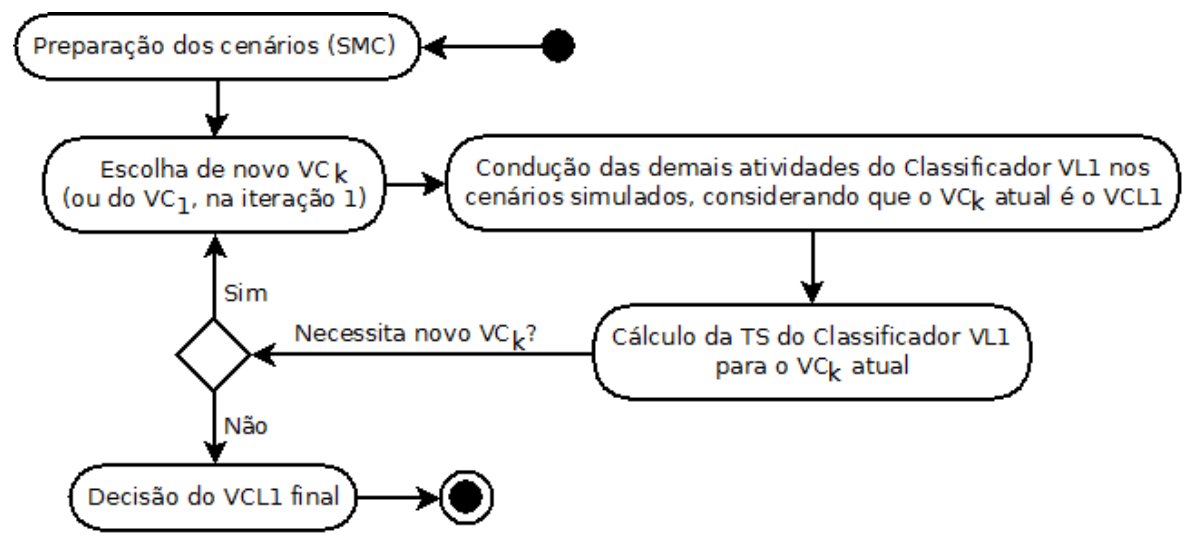

Figura 2. Diagrama de atividades para construção do VCL1. 
A aplicação do Classificador VL1 na identificação de outliers foi avaliada por comparação com o DS, em relação a cenários de redes nos quais os outliers eram conhecidos. A comparação entre os métodos, em ambos os casos, foi feita pelas respectivas TS.

\section{AP-MMQ}

Seja $|\mathbf{v}|$ o vetor do valor absoluto dos resíduos, e Ex e Ev o das raízes quadradas dos elementos da diagonal principal das MVCs (que representam as precisões) dos parâmetros estimados e dos resíduos no ajustamento pelo MMQ, respectivamente. Via SMC, para verificação da homogeneidade dos resultados, os desvios padrão dos elementos dos vetores $|\mathbf{v}|, \mathbf{E x}$ e Ev, após ajustamento das redes sem outliers pelo MMQ com o modelo estocástico usual dos pesos proporcionais ao inverso do comprimento das linhas de nivelamento, e com o modelo estocástico alternativo advindo do MRA, foram comparados.

\section{Dados}

Para consecução dos experimentos, observações reais da RAAP e cenários obtidos por SMC a partir de uma rede matemática foram utilizados.

\section{Rede Matemática - base para SMC}

As observações da rede matematicamente "perfeita" correspondem propositalmente aos desníveis exatos entre as respectivas RRNN. Trata-se de uma rede sem qualquer erro de medida (nem mesmo aleatórios), útil para aplicação de SMC, assim como feito por (Hekimoglu \& Erenoglu, 2007). Nas simulações a partir dela, a altitude do ponto A é considerada fixa e com valor $\mathrm{h}_{\mathrm{A}}=0$. Assim, foram simuladas redes com 20 observações e 10 pontos de altitudes a determinar (incógnitas), ou seja, 10 graus de liberdade. A geometria das redes simuladas consta da Figura 3.

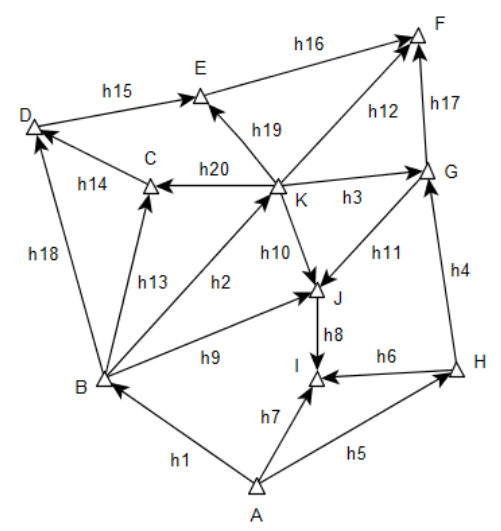

Figura 3. Geometria da rede matemática e das simulações decorrentes. 
Foi intitulada Simulação 1 a criação, a partir da Rede Matemática, de cenários aleatórios de rede altimétrica sem outliers. As observações foram simuladas da mesma forma que as "observations without outliers" do caso de heterocedasticidade de (Hekimoglu \& Erenoglu, 2007), com apenas uma diferença: como no corrente artigo se adotou a regra dos $3 \sigma$ para o erro total (Suraci \& Oliveira, 2019b) na definição de outlier, valores de erro aleatório com magnitude superior aos $3 \sigma$ não foram considerados. Nas simulações a partir da Rede Matemática, o desvio padrão das observações foi dado pela Equação 7, sendo K (em km) o comprimento da respectiva linha.

$$
\sigma_{i}=1,0(m m) * \sqrt{K_{i}}
$$

Já a Simulação 2 correspondeu à criação, a partir da Rede Matemática, de cenários aleatórios de rede altimétrica com outliers. As "boas" observações foram simuladas assim como na Simulação 1, e a simulação de outliers foi inspirada na das "bad observations" com sinal aleatório de (Hekimoglu \& Erenoglu, 2007). Maiores detalhes em (Suraci, 2018).

\section{Rede real - trecho da $R A A P$}

Os dados da RAAP utilizados nos experimentos foram adaptados de (Beserra, 1952). Tratam-se dos dados do nivelamento geométrico de precisão do território brasileiro que tinha sido realizado até então, configurando 105 linhas de nivelamento (observações), com um comprimento total de 15.706,25 km, passando por diversos estados do território brasileiro. O retângulo envolvente correspondente a este nivelamento é apresentado na Figura 4. Um arquivo nomeado "Rede_real_geometria.pdf" e um nomeado "Rede_real_observacoes.pdf", nos quais a geometria, e os comprimentos e desníveis das observações dessa rede podem ser vistos com maior detalhamento, foram disponibilizados em (Suraci, 2019).

A altitude de uma das RRNN (da RRNN chamada 3L) foi considerada fixa (injunção fixa) e com valor $h_{3 \mathrm{~L}}=10,8190 \mathrm{~m}$. Com uma quantidade de pontos de altitude a determinar de 67 , o número de graus de liberdade é 38 . Não foram identificados outliers nessa rede pelo DS ( $\operatorname{com} \alpha=0,001$ ), adotando-se pesos das observações equivalentes ao inverso do comprimento das respectivas linhas, multiplicados por um fator de proporcionalidade $A=200$, como feito por (Beserra, 1952).

\section{Descrição dos experimentos e Resultados}

Os experimentos foram conduzidos no software Octave. As soluções dos ajustamentos pelas normas L1 e L $\infty$ foram feitas por programação linear, utilizando o método 


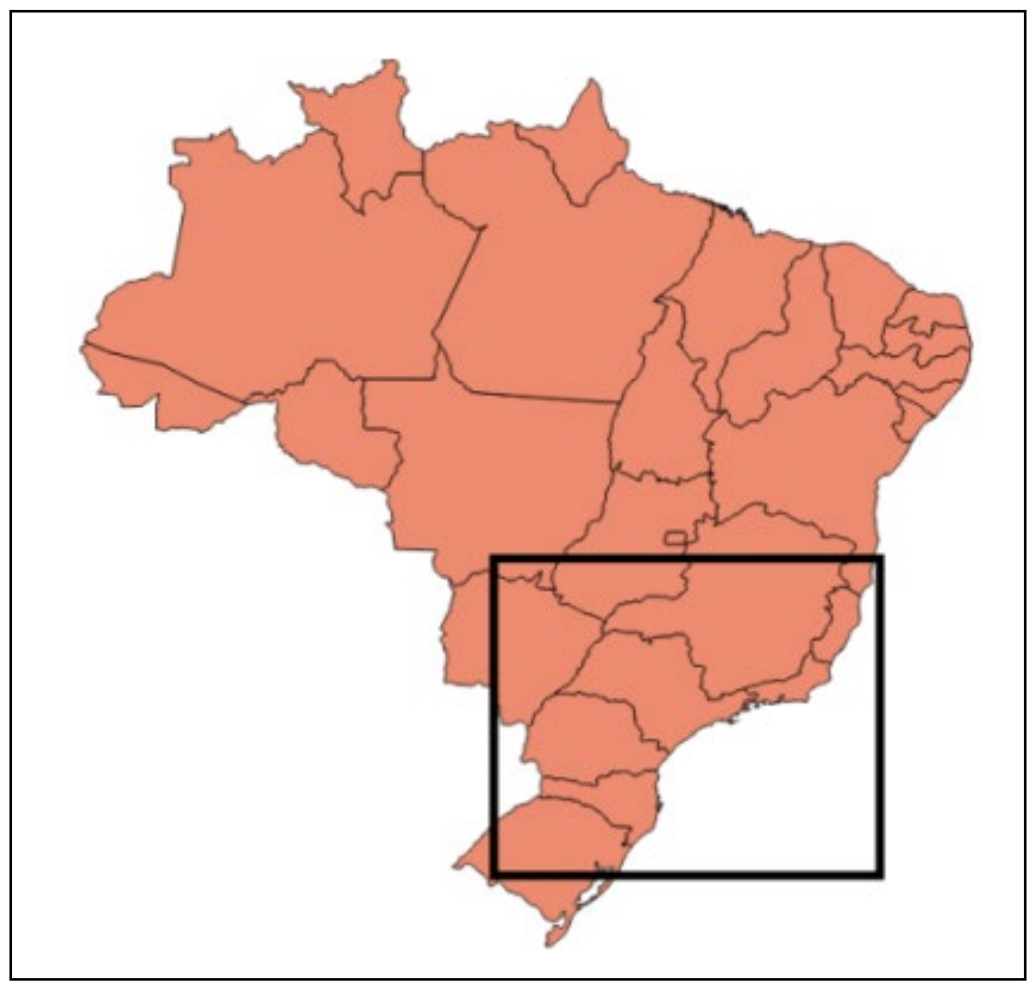

Figura 4. Retângulo envolvente da rede real.

SIMPLEx. O estado inicial das sequências de números pseudoaleatórios foi modificado sempre que necessário para evitar qualquer viés nos resultados. Os códigos dos experimentos estão disponibilizados em (Suraci, 2019).

\section{Experimento 1 - APPC-ML1}

No Experimento 1, a $A P P C-M L 1$ foi testada em cenários de rede obtidos pela Simulação 2. A Tabela 1 apresenta o PPCOMR (Equação 4) para diferentes intervalos de magnitude do erro do outlier propositalmente inserido. A linha "PIV" corresponde aos resultados dos ajustamentos pela ML1 com pesos pelo inverso da variância das respectivas observações (logo, proporcionais ao inverso do comprimento das linhas de nivelamento - Equação 7) e a "PUnit" com pesos unitários para as observações da rede. Já os parâmetros de comparação são indicados por "RA" para o resíduo absoluto e "RAP" para o resíduo absoluto ponderado. O total de cenários de rede simulados, para cada intervalo de magnitude de erro total inserido, foi de 200.000 . 
Tabela 1

PPCOMR - cenários com 1 outlier - Experimento 1

\begin{tabular}{cccccc}
\hline \multicolumn{2}{c}{ Erro Total } & $3-6 \sigma$ & $6-12 \sigma$ & $12-25 \sigma$ & $25-100 \sigma$ \\
\hline PUnit & RA & $\mathbf{6 3 , 7 9 \%}$ & $\mathbf{9 5 , 0 1 \%}$ & $\mathbf{9 9 , 9 2 \%}$ & $\mathbf{1 0 0 , 0 0 \%}$ \\
PIV & RA & $58,10 \%$ & $84,32 \%$ & $89,88 \%$ & $90,07 \%$ \\
PIV & RAP & $59,29 \%$ & $86,65 \%$ & $90,05 \%$ & $90,07 \%$ \\
\hline
\end{tabular}

A Tabela 2 é similar à Tabela 1 , mas para o caso de 2 outliers em cada cenário de rede simulado.

Tabela 2

PPCOMR - cenários com 2 outliers - Experimento 1

\begin{tabular}{lccccc}
\hline \multicolumn{2}{c}{ Erro Total } & $3-6 \sigma$ & $6-12 \sigma$ & $12-25 \sigma$ & $25-100 \sigma$ \\
\hline PUnit & RA & $\mathbf{3 8 , 0 2 \%}$ & $\mathbf{7 9 , 7 6 \%}$ & $\mathbf{8 9 , 3 7 \%}$ & $\mathbf{8 9 , 6 6 \%}$ \\
PIV & RA & $32,06 \%$ & $62,92 \%$ & $71,03 \%$ & $71,37 \%$ \\
PIV & RAP & $33,13 \%$ & $65,64 \%$ & $71,29 \%$ & $71,37 \%$ \\
\hline
\end{tabular}

Da análise das Tabelas 1 e 2, nota-se que o PPCOMR foi sempre maior no ajustamento pela ML1 com pesos unitários. Assim, verifica-se que a adoção de pesos unitários para as observações no ajustamento pela ML1 apresentou condições vantajosas para a identificação de outliers em relação aos usuais pesos proporcionais ao inverso do comprimento das linhas de nivelamento.

Vale notar ainda que a utilização de pesos usuais com o resíduo absoluto como parâmetro de comparação, abordagem conjunta comum na literatura, apresentou em geral o pior resultado (menor PPCOMR) dentre as três avaliadas. Ademais, as três abordagens conjuntas obtiveram aumento do PPCOMR para maiores magnitudes dos outliers inseridos e diminuição do PPCOMR com o aumento da quantidade de outliers.

\section{Experimento 2 - Classificador VL1 proposto}

O Experimento 2 visou comparar o desempenho do Classificador VL1 com o do DS na identificação de outliers. Para construção do VCL1, foram utilizados cenários de

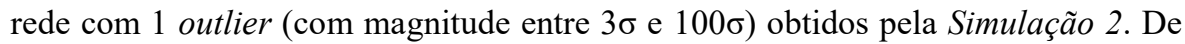
um total de 200.000 cenários simulados na identificação de outliers para cada um dos diversos VCs testados, o valor de 29,2 $\mathrm{mm}$ foi selecionado, por ter obtido a maior quantidade de acertos (191.307). Para cada cenário, foi considerado que houve acerto 
se o outlier foi identificado, sem que nenhuma outra observação também tenha sido assim classificada.

Deve-se atentar para o fato de que a construção do VCL1 ora apresentada foi feita com inserção de outliers entre $3 \sigma$ e $100 \sigma$, nos moldes da Simulação 2. A construção do VCL1 com inserção proposital de outliers com outros intervalos de magnitude, o que ocasionou diferentes valores para o VCL1 e, em consequência, diferentes TSs (Equação 6) para o Classificador VL1 nos experimentos a seguir, foi vista em (Suraci, 2018).

Na continuidade, o Classificador VL1, com o VCL1 construído, foi aplicado em cenários de rede com as diferentes magnitudes de erro dos outliers da Tabela 3, obtidos via Simulação 2. Para cada faixa de magnitude de outlier, foram testados 200.000 cenários simulados. A Tabela 3 apresenta a TS do Classificador VL1 e do DS nesse contexto.

Tabela 3

TS - 1 outlier - Experimento 2

\begin{tabular}{ccccc}
\hline Método & $3-6 \sigma$ & $6-12 \sigma$ & $12-25 \sigma$ & $25-100 \sigma$ \\
\hline DS $(\alpha=0,001)$ & $\mathbf{4 2 , 9 0 \%}$ & $\mathbf{9 3 , 9 2 \%}$ & $98,75 \%$ & $98,76 \%$ \\
Classificador VL1 & $18,99 \%$ & $79,02 \%$ & $\mathbf{9 8 , 9 3 \%}$ & $\mathbf{9 9 , 1 1 \%}$ \\
\hline
\end{tabular}

A Tabela 4 é similar à Tabela 3 , mas para o caso de 2 outliers inseridos propositalmente em cada cenário de rede simulado via Simulação 2. Nesse contexto, foi considerado que houve acerto na identificação de outliers se ambos foram identificados pelo método aplicado, sem que nenhuma outra observação também tenha sido assim classificada.

Tabela 4

TS - 2 outliers - Experimento 2

\begin{tabular}{|c|c|c|c|c|}
\hline Método & $3-6 \sigma$ & $6-12 \sigma$ & $12-25 \sigma$ & $25-100 \sigma$ \\
\hline $\operatorname{DS}(\alpha=0,001)$ & $14,32 \%$ & $76,55 \%$ & $87,29 \%$ & $88,88 \%$ \\
\hline Classificador VL1 & $3,35 \%$ & $56,34 \%$ & $87,90 \%$ & $88,32 \%$ \\
\hline
\end{tabular}

No caso anterior de 1 outlier, o DS apresentou tempo médio computacional inferior ao do Classificador VL1 (os tempos foram de 2,6 ms e 2,9 ms, respectivamente). Porém, seguindo para os testes com 2 outliers, o tempo médio do Classificador VL1 se manteve estável (2,9 ms), enquanto o do DS (4,2 ms) obteve uma tendência de crescimento, passando a ser maior que o do Classificador VL1. Isso era esperado, pois o DS é um método iterativo, enquanto o Classificador VL1 identifica os outliers em uma única iteração. 
As Tabelas 5 e 6 são similares à Tabela 4, mas tratando separadamente os casos de 2 outliers controláveis e de 2 não controláveis (considerando a análise da geometria da rede pelo MEM), respectivamente. Para o primeiro caso, a TS do DS foi sempre superior à do Classificador VL1. Entretanto, se ressalta o resultado do Classificador VL1 em cenários de dois outliers não controláveis, significativamente superior ao do DS.

Tabela 5

TS -2 outliers controláveis - Experimento 2

\begin{tabular}{ccccc}
\hline Método & $3-6 \sigma$ & $6-12 \sigma$ & $12-25 \sigma$ & $25-100 \sigma$ \\
\hline DS $(\alpha=0,001)$ & $\mathbf{1 6 , 0 1 \%}$ & $\mathbf{8 4 , 7 5 \%}$ & $\mathbf{9 5 , 9 9 \%}$ & $\mathbf{9 7 , 2 3 \%}$ \\
Classificador VL1 & $3,46 \%$ & $59,19 \%$ & $93,48 \%$ & $93,94 \%$ \\
\hline
\end{tabular}

Tabela 6

TS - 2 outliers não controláveis - Experimento 2

\begin{tabular}{ccccc}
\hline Método & $3-6 \sigma$ & $6-12 \sigma$ & $12-25 \sigma$ & $25-100 \sigma$ \\
\hline DS $(\alpha=0,001)$ & $1,24 \%$ & $19,39 \%$ & $27,07 \%$ & $31,36 \%$ \\
Classificador VL1 & $\mathbf{2 , 0 3 \%}$ & $\mathbf{3 4 , 5 6 \%}$ & $\mathbf{4 9 , 5 9 \%}$ & $\mathbf{4 9 , 7 1 \%}$ \\
\hline
\end{tabular}

Contudo, é lícito afirmar que o Experimento 2 mostrou que há cenários de redes altimétricas em que o Classificador VL1 foi mais eficaz que o DS na identificação de outliers, especialmente no caso de geometria da rede deficiente.

\section{Experimento $3-A P-M M Q$}

No Experimento 3, primeiramente a $A P-M M Q$ foi testada em 2.000 cenários de rede obtidos pela Simulação 1. O MRA médio calculado no ajustamento pela ML $\infty$ dos cenários simulados foi de $6,2162 \mathrm{~mm}$. O modelo estocástico alternativo foi obtido, em média, após 34 iterações do ajustamento pelo MMQ.

A Tabela 7 apresenta a quantidade de cenários nos quais o desvio padrão $(\sigma)$ dos elementos de $|\mathbf{v}|$, Ev e Ex foi menor (caracterizando maior homogeneidade dos elementos) para os ajustamentos pelo MMQ com modelo estocástico usual (ME usual) e com o modelo estocástico alternativo (ME alternativo) advindo do MRA.

\section{Tabela 7}

Quantidade de resultados mais homogêneos - Experimento 3 - Simulação 1

\begin{tabular}{ccc}
$\sigma$ & ME usual & ME alternativo \\
\hline$|\mathbf{v}|$ & 35 & $\mathbf{1 9 6 5}$ \\
$\mathbf{E v}$ & 153 & $\mathbf{1 8 4 8}$ \\
$\mathbf{E x}$ & 241 & $\mathbf{1 7 5 9}$ \\
\hline
\end{tabular}


Em seguida, a $A P-M M Q$ foi testada na Rede Real com dados da RAAP. A Tabela 8 apresenta os valores do desvio padrão dos elementos de $|\mathbf{v}|$, DPv e DPx para os ajustamentos pelo MMQ com o ME usual e com o ME alternativo. O MRA calculado no ajustamento pela ML $\infty$ para a rede foi de $0,1392 \mathrm{~m}$. O modelo estocástico alternativo foi obtido após 15 iterações. Vale esclarecer que o ajustamento pelo MMQ com o ME alternativo apresentou MRA (máximo elemento de $|\mathbf{v}|$ ) inferior em todos os casos (2.000 cenários simulados e rede com dados da RAAP). Obviamente, isso era de se esperar, pois esse modelo estocástico foi construído de forma a implicar isso.

\section{Tabela 8}

Resultados do ajustamento pelo MMQ - Experimento 3 - Trecho da RAAP

\begin{tabular}{ccc}
\hline$\sigma$ & ME usual & ME alternativo \\
\hline$|\mathbf{v}|$ & $0,0281 \mathrm{~m}$ & $\mathbf{0 , 0 2 3 8} \mathbf{~ m}$ \\
Ev & $0,0178 \mathrm{~m}$ & $\mathbf{0 , 0 0 4 2} \mathbf{~ m}$ \\
$\mathbf{E x}$ & $0,0248 \mathrm{~m}$ & $\mathbf{0 , 0 2 2 5} \mathbf{~ m}$ \\
\hline
\end{tabular}

Da análise da Tabela 7, verifica-se que o desvio padrão dos elementos dos vetores |v|, DPv e DPx com o ME alternativo foi menor que os equivalentes com o ME usual em cerca de $98 \%, 92 \%$ e $88 \%$ dos cenários simulados, respectivamente. Essa tendência também foi vista com os dados reais da RAAP (Tabela 8). Assim, na significativa maioria dos cenários analisados, o ajustamento pelo MMQ de redes altimétricas livres de outliers com modelagem estocástica alternativa advinda do MRA gerou resultados mais homogêneos que com o modelo usual.

A desvantagem do ME alternativo é que esta ponderação dificilmente reflete a realidade da rede. Neste caso o ajustamento das observações é obtido única e exclusivamente em função dos dados de campo e da configuração geométrica da rede. Logo, os resultados obtidos não necessariamente conduzem a uma solução ótima, uma vez que o modelo estocástico foi simplificado. Apesar do ME usual também representar uma simplificação, espera-se que ele seja uma aproximação mais realista.

Por fim, diante dos resultados, não se pode indicar qual dos modelos estocásticos deve ser seguido. Um modelo não exclui o outro. Contudo, os promissores resultados com o ME alternativo advindo do MRA mostram haver indícios da possibilidade de maiores investigações sobre o mesmo, ou até de formas de combinar os dois modelos estocásticos, visando resultados mais realistas e apropriados.

\section{Conclusões e implicações para a prática da estimação de redes altimétricas}

O Classificador VL1 proposto apresentou TS significativamente superior à do DS em cenários de outliers não controláveis (considerando o MEM). Isso é particularmente relevante para redes com grande quantidade de observações, mas baixa redundância 
função da geometria mais pobre, caso da RAAP e das redes de nivelamento de forma geral. A implantação de extensas redes altimétricas com geometria que permita grande resistência contra múltiplos outliers possui custo elevado, de forma que métodos com maior eficácia em cenários de menor resistência são de grande valia.

O tempo de execução computacional do Classificador VL1 se mantém estável com o aumento da quantidade de outliers nas observações, enquanto o do DS tende a ser aumentado, por ser esse último um método iterativo. Isso representa também uma vantagem do Classificador VL1 para cenários com quantidade relativamente grande de observações, como redes de nivelamento a nível nacional.

Quanto à aplicação da norma $\mathrm{L} \infty$, o modelo estocástico alternativo gerou resíduos e precisão desses e dos parâmetros estimados mais homogêneos, com menor desvio padrão, que aqueles com a abordagem usual para o modelo estocástico, na significativa maioria dos casos analisados. Isso pode ser aproveitado para que se evitem redes com vértices relativamente muito menos precisos que outros, especialmente quando o controle é baixo, algo também comum em redes de nivelamento.

Este artigo é apresentado a partir dos resultados da Dissertação de Mestrado intitulada: "Aplicação das normas L1 e L $\infty$ em redes altimétricas: identificação de outliers e construção do modelo estocástico", defendida em 19 de dezembro de 2018.

\section{Bibliografia}

Abdelmalek, N. \& Malek, W. (2008). Numerical linear approximation in C. CRC Press: Boca Raton, EUA, Taylor \& Francis Group.

Amiri-Simkooei, A. (2003). "Formulation of L1 Norm Minimization in GaussMarkov Models", Journal of Surveying Engineering, fev., pp. 37-43. https://doi.org/10.1061/(ASCE)0733-9453(2003)129:1(37)

Amiri-Simkooei, A. (2018). "On the use of two L1 norm minimization methods in geodetic networks", Earth Observation and Geomatics Engineering, 2(1): 1-8. https://doi.org/10.22059/EOGE.2018.256034.1021

Baarda, W. (1968). "A testing procedure for use in geodetic networks", Publications on Geodesy - New Series, 2(5): 1-97. Disponível em http://www.ncgeo.nl/downloads/09Baarda.pdf

Baselga, S. (2007). "Global Optimization Solution of Robust Estimation”, Journal of Surveying Engineering, 133(3): 123-128.

https://doi.org/ 10.1061/(ASCE)0733-9453(2007)133:3(123)

Beserra, H. (1952). Ajustamento da rede de nivelamento, IBGE, Rio de Janeiro, Brasil, Biblioteca Geográfica Brasileira.

Dantzig, G. (1963). Linear Programming and Extensions, The Rand Corporation, Santa Monica, EUA, Princeton University Press. 
Durdag, U.M., Hekimoglu, S. \& Erdogan, B. (2016). “Outlier detection by using fault detection and isolation techniques in geodetic networks", Survey Review, 48(351): 400-408. https://doi.org/10.1179/1752270615Y.0000000038

Ebong, M.B. (1986). "The Chebyshev adjustment of a geodetic levelling network", Survey Review, 28(220): p. 315-321. https://doi.org/10.1179/sre.1986.28.220.315

Gemael, C., Machado, A.M.L. \& Wandresen, R. (2015). Introdução ao ajustamento de observações: aplicações geodésicas, (2). UFPR, Curitiba, Brasil, Ed. UFPR.

Ghilani, C. (2010). Adjustment Computations: Spatial Data Analysis, (5), John Wiley \& Sons, Hoboken, EUA.

Hekimoglu, S., Erenoglu, R.C., Sanli, D.U. \& Erdogan, B. (2011). "Detecting Configuration Weaknesses in Geodetic Networks", Survey Review, 43(323): 713730. https://doi.org/10.1179/003962611X13117748892632

Hekimoglu, S. \& Erenoglu, R.C. (2007). "Effect of heteroscedasticity and heterogeneousness on outlier detection for geodetic networks", Journal of Geodesy, 81: 137-148. https://doi.org/10.1007/s00190-006-0095-z

IAG (2015). IAG Resolution (No. 1/2015) for the definition and realization of an International Height Reference System. Disponível em

https://ihrs.dgfi.tum.de/fileadmin/JWG 2011/IAG Resolutions 2015.pdf

IBGE (2018). Reajustamento da Rede Altimétrica com Números Geopotenciais 2018, IBGE, Rio de Janeiro, Brasil. Disponível em https://biblioteca.ibge.gov.br/visualizacao/livros/liv101666.pdf

Kampmann, G. \& Krause, B. (1997). “A breakdown point analysis for the straight line fit based on balanced observations", Bollettino di Geodesia e Scienze Affini, 3: 293-303.

Khodabandeh, A. \& Amiri-Simkooei, A. (2011). "Recursive Algorithm for L1 Norm Estimation in Linear Models", Journal of Surveying Engineering, 137(1): 1-8. https://doi.org/10.1061/(ASCE)SU.1943-5428.0000031

Klein, I., Matsuoka, M.T., Guzatto, M.P., Souza, S.F. \& Veronez, M.R., (2015). “On evaluation of different methods for quality control of correlated observations", Survey review, 47(340): 28-35.

https://doi.org/10.1179/1752270614Y.0000000089

Koch, I.E., Klein, I., Gonzaga Jr., L., Matsuoka, M.T., Rofatto, V.F., Veronez, M.R. (2019). "Robust Estimators in Geodetic Networks Based ona New Metaheuristic: Independent Vortices Search", Sensors, 19(20), 4535, pp. 1-27. https://doi.org/10.3390/s19204535

Koch, K. (1999). Parameter Estimation and Hypothesis Testing in Linear Models, (2), Springer, Berlim, Alemanha.

Marshall, J. \& Bethel, J. (1996). Basic concepts of L1 norm minimization for surveying applications, Journal of Surveying Engineering, 122(4): 168-179. https://doi.org/10.1061/(ASCE)0733-9453(1996)122:4(168) 
Mason, J.C. \& Handscomb, D.C., Chebyshev polynomials, CRC Press, Florida, EUA, 2003.

Raychaudhuri, S. (2008). "Introduction to Monte Carlo Simulation", Proceedings of the 2008 Winter Simulation Conference, pp. 91-100. Disponível em https://dl.acm.org/citation.cfm?id=1516768

Rofatto, V., Matsuoka, M. \& Klein, I. (2017). An Attempt to Analyse Baarda's Iterative Data Snooping Procedure based on Monte Carlo Simulation. South African Journal of Geomatics, 6(3): 416-435. Disponível em http://www.sajg.org.za/index.php/sajg/article/view/578

Rofatto, V.F., Matsuoka, M.T., Klein, I., Veronez, M.R., Bonimani, M.L. \& Lehmann, R. (2018). “A half-century of Baarda's concept of reliability: a review, new perspectives, and applications", Survey Review, https://doi.org/10.1080/00396265.2018.1548118

Suraci, S.S. (2018). Aplicação das normas L1 e Lo em redes altimétricas: identificação de outliers e construção do modelo estocástico, Dissertação Mestrado em Engenharia Cartográfica, IME: Rio de Janeiro, Brasil. Disponível em https://1drv.ms/b/s!AlQm3 WMI4DY1gdNKKtQ5NTnu7PQUQg

— (2019). "GitHub de Stefano Sampaio Suraci”, Disponível em https://github.com/stefano998/artigoRevCartografica

Suraci, S.S. \& Oliveira, L.C. (2019a). "Chebyshev norm minimization: what to expect? Case study in a leveling network", Revista Brasileira de Geomática: no prelo.

(2019b). "Outlier=gross error? Do only Gross errors cause outliers in geodetic networks? Addressing these and other questions", Boletim de Ciências Geodésicas, 25: 1-16. https://doi.org/10.1590/s1982-21702019000s00004

(2019c). "Proposta de modelo estocástico para minimização do máximo resíduo absoluto em redes de nivelamento", Revista Militar de Ciência e Tecnologia, 36: 55-58. Disponível em

http://ebrevistas.eb.mil.br/index.php/CT/article/view/2722

Suraci, S.S., Oliveira, L.C. \& Klein, I., (2019). "Two aspects on L1-norm adjustment of leveling networks", Revista Brasileira de Cartografia, 71(2): 486-500. https://doi.org/10.14393/rbcv71n2-47697

Teunissen, P., (2006). Testing theory: an introduction, (2), Delft Univ. of Technology: Delft, Holanda.

Yetkin, M. \& Inal, C. (2011). L1 norm minimization in GPS networks. Survey Review, 43(323): 523-532.

https://doi.org/10.1179/003962611X13117748892038 